\title{
Analytical expressions for the deprojected Sérsic model
}

\section{General expressions in terms of the Fox $\boldsymbol{H}$ function}

\author{
M. Baes and E. Van Hese
}

\begin{abstract}
Sterrenkundig Observatorium, Universiteit Gent, Krijgslaan 281 S9, 9000 Gent, Belgium
e-mail: maarten.baes@ugent.be
\end{abstract}

Received 15 July 2011 / Accepted 8 August 2011

\begin{abstract}
The Sérsic model is the de facto standard to describe the surface brightness distribution of hot stellar systems. An important inconvenience of this analytical model is that the corresponding luminosity density and associated properties cannot be expressed using elementary functions or even standard special functions. We present a set of compact and elegant analytical expressions for the luminosity density, cumulative luminosity, and potential for the Sérsic model in terms of the Fox $H$ function for general values of the Sérsic index. Furthermore, we present explicit series expansions of these quantities and discuss the asymptotic behaviour. Our analysis completes earlier work and demonstrates the power of the underestimated Fox $H$ function as a tool for analytical work.
\end{abstract}

Key words. methods: analytical - galaxies: photometry

\section{Introduction}

The Sérsic (1968) surface brightness profile has become the preferred model for describing the surface brightness profile of earlytype galaxies and the bulges of spiral galaxies (e.g. Davies et al. 1988; Caon et al. 1993; D’Onofrio et al. 1994; Cellone et al. 1994; Andredakis et al. 1995; Prugniel \& Simien 1997; Möllenhoff \& Heidt 2001; Graham \& Guzmán 2003; Allen et al. 2006; Gadotti 2009). Many analytical properties of this model have been discussed in the literature (e.g. Ciotti 1991; Ciotti \& Lanzoni 1997; Ciotti \& Bertin 1999; Trujillo et al. 2001; Mazure \& Capelato 2002; Cardone 2004; Graham \& Driver 2005; Elíasdóttir \& Möller 2007; Baes \& Gentile 2011).

An important inconvenience of the Sérsic model is that its deprojected luminosity density, i.e. the spatial 3D luminosity density $v(r)$ that projects on the plane of the sky to the Sérsic surface brightness profile, cannot be expressed using elementary functions or even in terms of standard special functions. It was long thought that no analytical expression could be obtained. Quite unexpectedly, Mazure \& Capelato (2002) came up with an analytical expression for $v(r)$ in terms of the Meijer $G$ function for all integer Sérsic indices $m$. Baes \& Gentile (2011, hereafter BG11) took this analysis one step further and showed that the deprojection of the Sérsic surface brightness profile for general values of $m$ can be solved elegantly using Mellin integral transforms and gives rise to a Mellin-Barnes integral. The result is that the Sérsic luminosity density can be written compactly in terms of a Fox $H$ function, which reduces to a Meijer $G$ function for all rational values of $m$. BG11 also calculated a number of additional properties of the Sérsic model for rational $m$, including the asymptotic expansion of the luminosity density at small and large radii, the cumulative light profile, and the gravitational potential. This was possible thanks to the many analytical properties of the Meijer $G$ function.

The goal of this paper is two-fold. Foremost, we extend and complete the analysis presented in Mazure \& Capelato (2002) and BG11. We provide compact and elegant expressions for the density, potential, and luminosity profiles in terms of the general Fox $H$ function, which are valid for all values of the Sérsic index $m$ rather than just for integer or rational $m$. We also present a completely general series expansion of these functions that enables both a numerical evaluation and a straightforward analytical study of the asymptotic behaviour. Besides providing these useful characteristics on one of the most used models in extragalactic astronomy, our work also has a secondary objective, namely to demonstrate the power of the Fox $H$ function for analytical work. The Fox $H$ function is, to use an understatement, not the most mainstream special function: it is even not contained in the standard works on special functions such as Gradshteyn \& Ryzhik (1965) or the Wolfram Functions Site. We feel that this is not justified because it is in fact a very elegant and powerful tool for analytical work, and it is becoming used more and more in mathematics and applied sciences, including physics, biology, engineering and earth sciences. It is also gradually being used in astrophysics, e.g. for solar and stellar structure models, fractional reaction-diffusion equations, and stellar dynamics (Haubold et al. 2007, 2011; Baes \& van Hese 2007; van Hese et al. 2009; De Rijcke et al. 2010). With our analysis, we wish to illustrate its useful properties and advocate its use in theoretical astrophysical research.

In Sect. 2 we derive compact expressions for the luminosity density, cumulative luminosity and potential for the Sérsic model, and in Sect. 3 we deduce detailed power and logarithmic-power series expressions for these important quantities. We discuss the asymptotic behaviour of our functions in Sect. 4, and we sum up our results in Sect. 5. In Appendix A we present the Fox $H$ function and we discuss some of its properties that were used for our analysis. 


\section{Analytical properties of the Sérsic model}

The Sérsic model is defined by the intensity profile projected on the plane of the sky,

$I(R)=I_{0} \exp \left[-b\left(\frac{R}{R_{\mathrm{e}}}\right)^{1 / m}\right]$

The 3D, deprojected luminosity density $v(r)$ of a spherically symmetric system can be recovered from the surface brightness profile $I(R)$ using the standard deprojection formula

$v(r)=-\frac{1}{\pi} \int_{r}^{\infty} \frac{\mathrm{d} I}{\mathrm{~d} R}(R) \frac{\mathrm{d} R}{\sqrt{R^{2}-r^{2}}}$.

Substituting the Sérsic profile (1) into (2) we obtain an integral that cannot readily be evaluated using the standard methods or look-up tables. BG11 applied a Mellin integral transform technique to convert this integral to a Mellin-Barnes contour integral,

$v(r)=\frac{2 m I_{0}}{\sqrt{\pi}} r^{-1} \frac{1}{2 \pi i} \int_{\mathcal{L}} \frac{\Gamma(2 m x) \Gamma\left(\frac{1}{2}+x\right)}{\Gamma(x)}\left(\frac{b^{m} r}{R_{\mathrm{e}}}\right)^{-2 x} \mathrm{~d} x$,

or, given the definition (A.1), to the compact expression

$v(r)=\frac{2 m I_{0} b^{m}}{\sqrt{\pi} R_{\mathrm{e}}} u^{-1} H_{1,2}^{2,0}\left[\begin{array}{c}(0,1) \\ (0,2 m),\left(\frac{1}{2}, 1\right)\end{array} \mid u^{2}\right]$,

where we have used the reduced coordinate

$u=\frac{b^{m} r}{R_{\mathrm{e}}}$.

As a check on this formula, BG11 calculated the total luminosity of the Sérsic model for rational $m$ by integrating the luminosity density over the entire space. To obtain the results, they used the integration properties of the Meijer $G$ function, combined with several applications of Gauss' multiplication theorem. As a generalization of this result, and as a nice example of the power of the Fox $H$ function, we calculate the total luminosity from the general formula (4), i.e.

$L=4 \pi \int_{0}^{\infty} v(r) r^{2} \mathrm{~d} r=\frac{4 m \sqrt{\pi} I_{0} R_{\mathrm{e}}^{2}}{b^{2 m}} \int_{0}^{\infty} H_{1,2}^{2,0}\left[\begin{array}{c}(0,1) \\ (0,2 m),\left(\frac{1}{2}, 1\right)\end{array} \mid t\right] \mathrm{d} t$.

To evaluate this integral, one should remind that equation (A.1) defined the Fox $H$ function as an inverse Mellin transform of a combination of gamma functions. As a result, the Mellin transform of a Fox $H$ function reads

$\left.\int_{0}^{\infty} H_{p, q}^{m, n}\left[\begin{array}{c}(\boldsymbol{a}, \boldsymbol{A}) \\ (\boldsymbol{b}, \boldsymbol{B})\end{array}\right] z\right] z^{s-1} \mathrm{~d} z=\frac{\prod_{j=1}^{m} \Gamma\left(b_{j}+B_{j} s\right) \prod_{j=1}^{n} \Gamma\left(1-a_{j}-A_{j} s\right)}{\prod_{j=m+1}^{q} \Gamma\left(1-b_{j}-B_{j} s\right) \prod_{j=n+1}^{p} \Gamma\left(a_{j}+A_{j} s\right)}$.

Applying this to (6) with $s=1$, we obtain

$L=\frac{4 m \sqrt{\pi} I_{0} R_{\mathrm{e}}^{2}}{b^{2 m}} \frac{\Gamma(2 m) \Gamma\left(\frac{3}{2}\right)}{\Gamma(1)}=\frac{\pi I_{0} R_{\mathrm{e}}^{2} \Gamma(2 m+1)}{b^{2 m}}$,

in agreement with the value obtained by integrating the surface brightness profile (1) over the plane of the sky.

From the luminosity density, a number of other important quantities can be derived, most importantly the cumulative luminosity profile $L(r)$ and the gravitational potential $\Psi(r)$,

$L(r)=4 \pi \int_{0}^{r} \rho\left(r^{\prime}\right) r^{\prime 2} \mathrm{~d} r^{\prime}$

$\Psi(r)=G \Upsilon \int_{r}^{\infty} \frac{L\left(r^{\prime}\right) \mathrm{d} r^{\prime}}{r^{\prime 2}}$

where the $\Upsilon$ is the mass-to-light ratio. Mazure \& Capelato (2002) and BG11 calculated these quantities for the Sérsic model for integer and rational values of the Sérsic parameter, respectively, using the integration properties of the Meijer $G$ function. It is, however, possible to calculate these properties for general $m$ in an elegant way by directly applying the integrations on the MellinBarnes integral form of the luminosity density. For the cumulative luminosity profile we find

$$
\begin{aligned}
L(r) & =8 m \sqrt{\pi} I_{0} \int_{0}^{r}\left[\frac{1}{2 \pi i} \int_{\mathcal{L}} \frac{\Gamma(2 m x) \Gamma\left(\frac{1}{2}+x\right)}{\Gamma(x)}\left(\frac{b^{m} r^{\prime}}{R_{\mathrm{e}}}\right)^{-2 x} \mathrm{~d} x\right] r^{\prime} \mathrm{d} r^{\prime} \\
& =8 m \sqrt{\pi} I_{0} \frac{1}{2 \pi i} \int_{\mathcal{L}} \frac{\Gamma(2 m x) \Gamma\left(\frac{1}{2}+x\right)}{\Gamma(x)}\left(\frac{b^{m}}{R_{\mathrm{e}}}\right)^{-2 x}\left[\int_{0}^{r} r^{\prime 1-2 x} \mathrm{~d} r^{\prime}\right] \mathrm{d} x \\
& =4 m \sqrt{\pi} I_{0} r^{2} \frac{1}{2 \pi i} \int_{\mathcal{L}} \frac{\Gamma(2 m x) \Gamma\left(\frac{1}{2}+x\right) \Gamma(1-x)}{\Gamma(x) \Gamma(2-x)} u^{-2 x} \mathrm{~d} x \\
& =\frac{4 m \sqrt{\pi} I_{0} R_{\mathrm{e}}^{2}}{b^{2 m}} u^{2} H_{2,3}^{2,1}\left[\begin{array}{c}
(0,1),(0,1) \\
(0,2 m),\left(\frac{1}{2}, 1\right),(-1,1)
\end{array} \mid u^{2}\right] .
\end{aligned}
$$


For the gravitational potential we find after a similar calculation

$\Psi(r)=\frac{2 m \sqrt{\pi} G \Upsilon I_{0} R_{\mathrm{e}}}{b^{m}} u H_{2,3}^{2,1}\left[\begin{array}{c}(0,1),(0,1) \\ (0,2 m),\left(-\frac{1}{2}, 1\right),(-1,1)\end{array} \mid u^{2}\right]$.

The formulae (4), (11), and (12) form a triplet of formulae that describe three important spatial properties of the Sérsic model in a compact way.

A straightforward way of checking these formulae is to look at the model that corresponds to $m=\frac{1}{2}$. In this case, all components of the vectors $\boldsymbol{A}$ and $\boldsymbol{B}$ are equal to one, such that the Fox $H$ function reduces to a Meijer $G$ function. We find

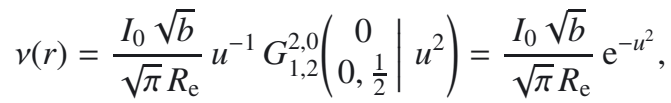

$L(r)=\frac{2 \sqrt{\pi} I_{0} R_{\mathrm{e}}^{2}}{b} u^{2} G_{2,3}^{2,1}\left(\begin{array}{c}0,0 \\ 0, \frac{1}{2},-1\end{array} \mid u^{2}\right)=\frac{\pi I_{0} R_{\mathrm{e}}^{2}}{b}\left[\operatorname{erf} u-\frac{2}{\sqrt{\pi}} u \mathrm{e}^{-u^{2}}\right]$,

$\Psi(r)=\frac{\sqrt{\pi} G^{\Upsilon} I_{0} R_{\mathrm{e}}}{\sqrt{b}} u G_{2,3}^{2,1}\left(\begin{array}{c}0,0 \\ 0,-\frac{1}{2},-1\end{array} \mid u^{2}\right)=\frac{\pi G \Upsilon I_{0} R_{\mathrm{e}}}{\sqrt{b}} \frac{\operatorname{erf} u}{u}$.

These expressions can also be derived by substituting the intensity profile $I(R)=I_{0} \mathrm{e}^{-b R^{2} / R_{\mathrm{e}}^{2}}$ in the expressions (2), (9), and (10) and directly evaluating the resulting integrals. More generally, one can check that the formulae (4), (11), and (12) reduce to Eqs. (22), (40), and (44) of BG11 for rational values of $m$.

\section{Explicit series expansions}

While the expressions (4), (11), and (12) form an triplet of compact formulae that are useful for analytical work, they are not readily useful to numerically evaluate the spatial properties of the Sérsic model. For rational values of $m$, the Fox $H$ functions reduce to Meijer $G$ functions, and some numerical software packages now have this function implemented. However, the numerical evaluation of Meijer $G$ functions with large parameter vectors (which easily occurs in our case for rational values of $m$, as can be seen in BG11), proves to be difficult, in particular in cases where second-order poles are present in the integrand of the inverse Mellin transform. Moreover, for general values of $m$, the expressions (4), (11), and (12) cannot be written in terms of the Meijer $G$ function or any other special function, and we are not aware of any implementations in numerical software that can evaluate general Fox $H$ functions.

In this section, we derive explicit series expansions for $v(r), L(r)$, and $\Psi(r)$, which both enables the numerical calculation and again highlights the power of Fox $H$ function as a useful mathematical tool. The expansions build on the general series expansion of the Fox $H$ function as power or power-logarithmic series (details can be found in Appendix A). The form of the series expansion depends on the multiplicity of the poles of the gamma functions $\Gamma\left(b_{j}+B_{j} s\right)$. For $v(r)$ and $L(r)$, the poles of these gamma functions are found at $-k_{1} / 2 m$ and $-1 / 2-k_{2}$ with $k_{1}$ and $k_{2}$ any natural number. The gamma functions corresponding to the expression of the potential $\Psi(r)$ contain the same poles with an additional pole at $1 / 2$. The good news is that each pole can at most occur twice, the bad news is that this happens quite often: for all integer $m$ and rational $m=p / q$ where the denominator $q$ of the fraction is odd, double poles do occur.

In the case of simple poles of the gamma functions $\Gamma\left(b_{j}+B_{j} s\right)$, the expansion of the Fox $H$ function is a power series, given by Eq. (A.6). Applied to our case, we find in case $m$ is non-rational or rational with an even denominator, the expansions

$$
\begin{aligned}
& v(r)=\frac{2 m I_{0} b^{m}}{\sqrt{\pi} R_{\mathrm{e}}}\left[\sum_{k=1}^{\infty} \frac{\Gamma\left(\frac{1}{2}-\frac{k}{2 m}\right)}{\Gamma\left(-\frac{k}{2 m}\right)} \frac{(-1)^{k}}{k !} \frac{u^{k / m-1}}{2 m}+\sum_{k=0}^{\infty} \frac{\Gamma(-m-2 m k)}{\Gamma\left(-\frac{1}{2}-k\right)} \frac{(-1)^{k}}{k !} u^{2 k}\right], \\
& L(r)=\frac{4 m \sqrt{\pi} I_{0} R_{\mathrm{e}}^{2}}{b^{2 m}}\left[\sum_{k=1}^{\infty} \frac{\Gamma\left(\frac{1}{2}-\frac{k}{2 m}\right)}{\Gamma\left(-\frac{k}{2 m}\right)} \frac{(-1)^{k}}{k !} \frac{u^{k / m+2}}{k+2 m}+\sum_{k=0}^{\infty} \frac{2 \Gamma(-m-2 m k)}{\Gamma\left(-\frac{1}{2}-k\right)} \frac{(-1)^{k}}{k !} \frac{u^{2 k+3}}{2 k+3}\right], \\
& \Psi(r)=\frac{2 m \sqrt{\pi} G \Upsilon I_{0} R_{\mathrm{e}}}{b^{m}}\left[\sum_{k=1}^{\infty} \frac{\Gamma\left(-\frac{1}{2}-\frac{k}{2 m}\right)}{\Gamma\left(-\frac{k}{2 m}\right)} \frac{(-1)^{k}}{k !} \frac{u^{k / m+1}}{k+2 m}+\sum_{k=0}^{\infty} \frac{2 \Gamma(m-2 m k)}{\Gamma\left(\frac{1}{2}-k\right)} \frac{(-1)^{k}}{k !} \frac{u^{2 k}}{2 k+1}\right] .
\end{aligned}
$$

The term $k=0$ in the first sums is omitted, since the factors $\Gamma(0)$ in the denominator make those terms vanish. In fact, if $m$ is a rational number $p / q$ with $q$ even, then the terms in the first sums for which $k=0, p, 2 p, \ldots$ vanish; if $p=1$, these first sums vanish completely. A particularly interesting case is (again) $m=\frac{1}{2}$, where we find

$$
\begin{aligned}
& v(r)=\frac{I_{0} \sqrt{b}}{\sqrt{\pi} R_{\mathrm{e}}} \sum_{k=0}^{\infty} \frac{(-1)^{k}}{k !} u^{2 k}=\frac{I_{0} \sqrt{b}}{\sqrt{\pi} R_{\mathrm{e}}} \mathrm{e}^{-u^{2}}, \\
& L(r)=\frac{4 \sqrt{\pi} I_{0} R_{\mathrm{e}}^{2}}{b} \sum_{k=0}^{\infty} \frac{(-1)^{k}}{k !} \frac{u^{3+2 k}}{2 k+3}=\frac{\pi I_{0} R_{\mathrm{e}}^{2}}{b}\left[\operatorname{erf} u-\frac{2}{\sqrt{\pi}} u \mathrm{e}^{-u^{2}}\right], \\
& \Psi(r)=\frac{2 \sqrt{\pi} G \Upsilon I_{0} R_{\mathrm{e}}}{\sqrt{b}} \sum_{k=0}^{\infty} \frac{(-1)^{k}}{k !} \frac{u^{2 k}}{2 k+1}=\frac{\pi G \Upsilon I_{0} R_{\mathrm{e}}}{\sqrt{b}} \frac{\operatorname{erf} u}{u},
\end{aligned}
$$

in agreement with formulae (13), (14) and (15). 
When the gamma functions $\Gamma\left(b_{j}+B_{j} s\right)$ have multiple poles, the expansion of the Fox $H$ function is a logarithmic-power series, the complexity of which increases with increasing multiplicity of the poles. The full expression for the case where two gamma functions share poles is given in Eq. (A.10). For our present case, this means that we obtain logarithmic-power series expansions for $v(r), L(r)$, and $\Psi(r)$ if the Sérsic index $m$ is integer or rational with an odd denominator. If we define $k_{0}=(q+1) / 2$, one obtains after quite some algebra

$$
\begin{aligned}
& v(r)=\frac{2 m I_{0} b^{m}}{\sqrt{\pi} R_{\mathrm{e}}}\left\{\sum_{\substack{k=1 \\
k \bmod p \neq 0}}^{\infty} \frac{\Gamma\left(\frac{1}{2}-\frac{k}{2 m}\right)}{\Gamma\left(-\frac{k}{2 m}\right)} \frac{(-1)^{k}}{k !} \frac{u^{k / m-1}}{2 m}+\sum_{\substack{k=0 \\
\left(k+k_{0}\right) \bmod }}^{\infty} \frac{\Gamma(-m-2 m k)}{\Gamma\left(-\frac{1}{2}-k\right)} \frac{(-1)^{k}}{k !} u^{2 k}\right. \\
& \left.-\frac{1}{\sqrt{\pi}} \sum_{\substack{k=0 \\
\left(k+k_{0}\right) \bmod }}^{\infty} \frac{(-1)^{p}}{2 m} \frac{(2 k+1) !}{(2 k m+m) ! k ! k !}\left(\frac{u}{2}\right)^{2 k}\left[-\ln \left(\frac{u}{2}\right)+\psi(k+1)+m \psi(2 k m+m)-\psi(2 k+1)\right]\right\}, \\
& L(r)=\frac{4 m \sqrt{\pi} I_{0} R_{\mathrm{e}}^{2}}{b^{2 m}}\left\{\sum_{\substack{k=1 \\
k \bmod p \neq 0}}^{\infty} \frac{\Gamma\left(\frac{1}{2}-\frac{k}{2 m}\right)}{\Gamma\left(-\frac{k}{2 m}\right)} \frac{(-1)^{k}}{k !} \frac{u^{k / m+2}}{k+2 m}+\sum_{\substack{k=0 \\
\left(k+k_{0}\right) \bmod q \neq 0}}^{\infty} \frac{2 \Gamma(-m-2 m k)}{\Gamma\left(-\frac{1}{2}-k\right)} \frac{(-1)^{k}}{k !} \frac{u^{2 k+3}}{2 k+3}\right. \\
& \left.-\frac{u^{3}}{\sqrt{\pi}} \sum_{\substack{k=0 \\
\left(k+k_{0}\right) \bmod q=0}}^{\infty} \frac{(-1)^{p}}{2 k m+3 m} \frac{(2 k+1) !}{(2 k m+m) ! k ! k !}\left(\frac{u}{2}\right)^{2 k}\left[-\ln \left(\frac{u}{2}\right)+\frac{1}{2 k+3}+\psi(k+1)+m \psi(2 k m+m)-\psi(2 k+1)\right]\right\}, \\
& \Psi(r)=\frac{2 m \sqrt{\pi} G \Upsilon I_{0} R_{\mathrm{e}}}{b^{m}}\left\{\sum_{\substack{k=1 \\
k \bmod p \neq 0}}^{\infty} \frac{\Gamma\left(-\frac{1}{2}-\frac{k}{2 m}\right)}{\Gamma\left(-\frac{k}{2 m}\right)} \frac{(-1)^{k}}{k !} \frac{u^{k / m+1}}{k+2 m}-\sum_{\substack{k=0 \\
\left(k+k_{0}\right) \bmod }}^{\infty} \frac{2 \Gamma(-m-2 m k)}{\Gamma\left(-\frac{1}{2}-k\right)} \frac{(-1)^{k}}{(k+1) !} \frac{u^{2 k+2}}{2 k+3}+\frac{2 \Gamma(m)}{\sqrt{\pi}}\right. \\
& \left.+\frac{u^{2}}{\sqrt{\pi}} \sum_{\substack{k=0 \\
\left(k+k_{0}\right) \bmod }}^{\infty} \frac{(-1)^{p}}{2 k m+3 m} \frac{(2 k+1) !}{(2 k m+m) ! k !(k+1) !}\left(\frac{u}{2}\right)^{2 k}\left[-\ln \left(\frac{u}{2}\right)+\frac{1}{2 k+2}+\frac{1}{2 k+3}+\psi(k+1)+m \psi(2 k m+m)-\psi(2 k+1)\right]\right\} .
\end{aligned}
$$

In these expressions, $\psi(s)=\Gamma^{\prime}(s) / \Gamma(s)$ is the digamma function. In $\Psi(r)$, the third term $2 \Gamma(m) / \sqrt{\pi}$ corresponds to the residue of the pole $\frac{1}{2}$. Again, the terms in the first sums for which $k=0, p, 2 p, \ldots$ vanish, and these first sums vanish completely if $p=1$. On the other hand, if $q=1$, i.e. if $m$ is an integer value, the second sums vanish, since then the integrands for $v(r)$ and $L(r)$ have no simple poles $\beta_{2, k_{2}}$, while for $\Psi(r)$ only the pole $\frac{1}{2}$ remains as a single pole. On the crossroad of these two cases we have $m=1$ : for this model, both the first and second sums in the expansions (22)-(24) vanish completely, apart from a single term for the potential. Astrophysically, the Sérsic model with $m=1$ corresponds to a model with an exponential surface brightness profile, often used for the description of low-luminosity elliptical galaxies and pseudo-bulges. BG11 calculated the luminosity density of the exponential model by directly deprojecting the surface brightness profile and through its representation as a Meijer $G$ function. For the luminosity density we get

$v(r)=\frac{I_{0} b}{\pi R_{\mathrm{e}}} \sum_{k=0}^{\infty} \frac{1}{k ! k !}\left(\frac{u}{2}\right)^{2 k}\left[-\ln \left(\frac{u}{2}\right)+\psi(k+1)\right]=\frac{I_{0} b}{\pi R_{\mathrm{e}}} K_{0}(u)$,

with $K_{v}(z)$ the modified Bessel function of the second kind. This expression agrees with Eq. (24) of BG11. Similarly, we obtain for the cumulative luminosity

$$
\begin{aligned}
L(r) & =\frac{4 I_{0} R_{\mathrm{e}}^{2}}{b^{2}} \sum_{k=0}^{\infty} \frac{8}{(2 k+3) k ! k !}\left(\frac{u}{2}\right)^{2 k+3}\left[-\ln \left(\frac{u}{2}\right)+\frac{1}{2 k+3}+\psi(k+1)\right] \\
& =\frac{2 \pi I_{0} R_{\mathrm{e}}^{2}}{b^{2}} u\left[K_{2}(u) L_{1}(u)+K_{1}(u) L_{2}(u)-\frac{4}{3 \pi} u K_{1}(u)\right],
\end{aligned}
$$

with $L_{v}(u)$ the modified Struve function. Finally, for the potential of the exponential model we get the expansion

$$
\begin{aligned}
\Psi(r) & =\frac{4 G \Upsilon I_{0} R_{\mathrm{e}}}{b}\left\{1-\sum_{k=0}^{\infty} \frac{2}{(2 k+3) k !(k+1) !}\left(\frac{u}{2}\right)^{2 k+2}\left[-\ln \left(\frac{u}{2}\right)+\frac{1}{2 k+3}+\frac{1}{2 k+2}+\psi(k+1)\right]\right\} \\
& =\frac{2 \pi G \Upsilon I_{0} R_{\mathrm{e}}}{b}\left[K_{2}(u) L_{1}(u)+K_{1}(u) L_{2}(u)+\frac{2}{3 \pi} u K_{1}(u)\right] .
\end{aligned}
$$

These last two expressions can also be obtained by substituting the luminosity density (25) into the recipes (9) and (10). 
M. Baes and E. Van Hese: Analytical expressions for the deprojected Sérsic model. II.

\section{Asymptotic behaviour}

With all the explicit power series of Sect. 3 available, it is fairly straightforward to examine the asymptotic behaviour of the spatial function of the Sérsic model at small radii, generalizing the results of BG11. The density has the following rich behaviour, depending on the value of $m$ :

$$
\begin{array}{ll}
v(r) \sim \frac{I_{0} b^{m}}{\pi R_{\mathrm{e}}}\left[\Gamma(1-m)+\frac{1}{2} \Gamma(1-3 m) u^{2}\right] & \text { for } 0<m<\frac{1}{3} \text { or } m=\frac{1}{2}, \\
v(r) \sim \frac{I_{0} b^{1 / 3}}{\pi R_{\mathrm{e}}}\left[\Gamma\left(\frac{2}{3}\right)-\frac{1}{2}\left(3 \ln \left(\frac{u}{2}\right)+\gamma+\frac{3}{2}\right) u^{2}\right] & \text { for } m=\frac{1}{3}, \\
v(r) \sim \frac{I_{0} b^{m}}{\pi R_{\mathrm{e}}}\left[\Gamma(1-m)+\frac{\sqrt{\pi}}{2 m} \frac{\Gamma\left(\frac{1}{2}-\frac{1}{2 m}\right)}{\Gamma\left(1-\frac{1}{2 m}\right)} u^{1 / m-1}\right] & \text { for } \frac{1}{3}<m<1 \text { and } m \neq \frac{1}{2}, \\
v(r) \sim \frac{I_{0} b}{\pi R_{\mathrm{e}}}\left[-\ln \left(\frac{u}{2}\right)-\gamma\right] & \text { for } m=1, \\
v(r) \sim \frac{I_{0} b^{m}}{\sqrt{\pi} R_{\mathrm{e}}} \frac{1}{2 m} \frac{\Gamma\left(\frac{1}{2}-\frac{1}{2 m}\right)}{\Gamma\left(1-\frac{1}{2 m}\right)} u^{1 / m-1} & \text { for } m>1,
\end{array}
$$

with $\gamma \approx 0.57721566$ the Euler-Mascheroni constant. The luminosity behaves as

$$
L(r) \sim \frac{4 I_{0} R_{\mathrm{e}}^{2}}{3 b^{2 m}} \Gamma(1-m) u^{3} \quad \text { for } m<1,
$$

$L(r) \sim \frac{4 I_{0} R_{\mathrm{e}}^{2}}{3 b^{2}}\left[-\ln \left(\frac{u}{2}\right)-\gamma+\frac{1}{3}\right] u^{3} \quad$ for $m=1$,

$L(r) \sim \frac{I_{0} R_{\mathrm{e}}^{2}}{b^{2 m}} \frac{2 \sqrt{\pi}}{2 m+1} \frac{\Gamma\left(\frac{1}{2}-\frac{1}{2 m}\right)}{\Gamma\left(1-\frac{1}{2 m}\right)} u^{1 / m+2} \quad$ for $m>1$

Finally, the potential approaches $r \rightarrow 0$ as

$$
\begin{array}{ll}
\Psi(r) \sim \frac{G \Upsilon I_{0} R_{\mathrm{e}}}{b^{m}}\left[4 \Gamma(1+m)-\frac{2}{3} \Gamma(1-m) u^{2}\right] & \text { for } m<1, \\
\Psi(r) \sim \frac{G \Upsilon I_{0} R_{\mathrm{e}}}{b}\left[4+\frac{2}{3}\left(\ln \left(\frac{u}{2}\right)+\gamma-\frac{5}{6}\right) u^{2}\right] & \text { for } m=1, \\
\Psi(r) \sim \frac{G \Upsilon I_{0} R_{\mathrm{e}}}{b^{m}}\left[4 \Gamma(1+m)+\frac{\sqrt{\pi}}{2 m+1} \frac{\Gamma\left(-\frac{1}{2}-\frac{1}{2 m}\right)}{\Gamma\left(1-\frac{1}{2 m}\right)} u^{1 / m+1}\right] & \text { for } m>1 .
\end{array}
$$

If we set $r=0$ in the expressions (30), we recover the central potential

$\Psi_{0}=\frac{4 G \Upsilon I_{0} R_{\mathrm{e}}}{b^{m}} \Gamma(m+1)$

for every real value $m>0$, in agreement with Eq. (12) of Ciotti (1991). 


\section{Conclusions}

This paper is the second paper that deals with the derivation of analytical expressions for the luminosity density $v(r)$, cumulative luminosity $L(r)$, and gravitational potential $\Psi(r)$ of the Sérsic model. Our work extends the work initiated by Mazure \& Capelato (2002), who managed to express these properties in terms of the Meijer $G$ function for integer values of the Sérsic index $m$. In BG11 we extended this analysis by demonstrating that these expressions can be derived using a Mellin integral transform and that they are also valid for half-integer values of $m$. We also derived more general expressions in terms of the Meijer $G$ function for these spatial properties for Sérsic models with rational $m$. Actually, the Mellin integral transform approach directly led to an expression in terms of the Fox $H$ function for the luminosity density valid for general values of $m$. Not fully aware of the rich power of the Fox $H$ function and attracted by the availability of implementations of the Meijer $G$ function both in symbolic computer algebra packages and as high-performance computing code, we focused our attention in BG11 on the study of the cases for rational $m$. In this paper, we have extended this analysis to arbitrary values of $m$, now making full use of the Fox $H$ function. We derived compact and elegant expressions for the luminosity density, cumulative luminosity, and gravitational potential that are valid for all values of $m$. We used the properties of the Fox $H$ function to deduce explicit power and logarithmic-power expansions for $v, L$, and $\Psi$, which can be used to evaluate these important spatial properties for arbitrary values of $m$. These series expansions also provide a direct way to probe the rich asymptotic behaviour of the Sérsic model at small radii, and our results are in full agreement with the more ad hoc analysis of BG11.

Our work fits into an impressive set of in-depth analytical work on the Sérsic model. Apart from the spatial properties discussed here, also its photometric (Graham \& Driver 2005), dynamic (Ciotti 1991; Ciotti \& Lanzoni 1997), and lensing (Cardone 2004; Elíasdóttir \& Möller 2007) properties have been studied extensively through analytical means. Given that the Sérsic model is the de facto standard model for describing the surface brightness distribution of hot stellar systems, this combined range of analytical properties has plenty of applications. For example, they can be used for the construction of self-consisting dynamical models for elliptical galaxies, serve as a realistic starting point for numerical $N$-body or smoothed particle hydrodynamics simulations, or serve as a template for radiative transfer or gravitational lens modelling.

Besides this practical use, we hope to have demonstrated the power of the Fox $H$ as a tool for analytical work. We are aware that the Fox $H$ function is not the most everyday special function and that its unfamiliar definition through inverse Mellin transform alone might already seem daunting. However, the Fox $H$ function is gradually appearing more in mathematics and applied sciences, and there are now several volumes of comprehensive literature available on its properties (e.g. Mathai \& Saxena 1978; Srivastava et al. 1982; Kilbas \& Saigo 2004; Mathai et al. 2009). Given the completeness and elegance of the analytical results obtained in this paper, we hope that this work may advocate the use of the Fox $H$ function in theoretical astrophysics.

\section{Appendix A: The Fox $\boldsymbol{H}$ function}

The Fox $H$ function, or just the $H$ function, is generally defined as the inverse Mellin transform of a product of gamma functions,

$\left.H_{p, q}^{m, n}\left[\begin{array}{c}(\boldsymbol{a}, \boldsymbol{A}) \\ (\boldsymbol{b}, \boldsymbol{B})\end{array}\right] z\right]=\frac{1}{2 \pi \mathrm{i}} \int_{\mathcal{L}} \frac{\prod_{j=1}^{m} \Gamma\left(b_{j}+B_{j} s\right) \prod_{j=1}^{n} \Gamma\left(1-a_{j}-A_{j} s\right)}{\prod_{j=m+1}^{q} \Gamma\left(1-b_{j}-B_{j} s\right) \prod_{j=n+1}^{p} \Gamma\left(a_{j}+A_{j} s\right)} z^{-s} \mathrm{~d} s$.

It was introduced by Fox (1961) in his attempt to find the most general symmetric Fourier kernel. The Fox $H$ function is a generalization of the Meijer $G$ function, but includes many other special functions, including Mittag-Leffler functions and generalized Bessel functions. For details on the definition, convergence, and many useful properties of the Fox $H$ function, we refer to Mathai \& Saxena (1978), Srivastava et al. (1982), Kilbas \& Saigo (2004), Mathai et al. (2009), and the references therein.

We now derive a series expansion of the Fox $H$ function. We write the Fox $H$ function in the form

$H_{p, q}^{m, n}\left[\begin{array}{c}(\boldsymbol{a}, \boldsymbol{A}) \\ (\boldsymbol{b}, \boldsymbol{B})\end{array} \mid z\right]=\frac{1}{2 \pi i} \int_{\mathcal{L}} \varphi(s) z^{-s} \mathrm{~d} s$

with

$\varphi(s)=\frac{\prod_{j=1}^{m} \Gamma\left(b_{j}+B_{j} s\right) \prod_{j=1}^{n} \Gamma\left(1-a_{j}-A_{j} s\right)}{\prod_{j=m+1}^{q} \Gamma\left(1-b_{j}-B_{j} s\right) \prod_{j=n+1}^{p} \Gamma\left(a_{j}+A_{j} s\right)}$.

Under certain conditions (always satisfied for the Fox $H$ functions we consider in this paper; see e.g. Kilbas \& Saigo 1999; or Mathai et al. 2009, for details), the Fox $H$ function is an analytical function, and the contour integral can be evaluated using the residue theorem. If we introduce the short-hand notation $\beta_{i, k}=-\left(b_{i}+k\right) / B_{i}$ for the poles of the functions $\Gamma\left(b_{i}+B_{i} s\right)$, then

$H_{p, q}^{m, n}\left[\begin{array}{c}(\boldsymbol{a}, \boldsymbol{A}) \\ (\boldsymbol{b}, \boldsymbol{B})\end{array} \mid z\right]=\sum_{i=1}^{m} \sum_{k=0}^{\infty} \operatorname{Res}_{s=\beta_{i, k}}\left[\varphi(s) z^{-s}\right]$

If $\beta_{i, k}$ is a simple pole, then the corresponding residue is fairly straightforward. We find

$\operatorname{Res}_{s=\beta_{i, k}}\left[\varphi(s) z^{-s}\right]=\lim _{s \rightarrow \beta_{i, k}}\left(s-\beta_{i, k}\right) \varphi(s) z^{-s}=\varphi_{i}\left(\beta_{i, k}\right) \frac{(-1)^{k}}{k !} \frac{z^{-\beta_{i, k}}}{B_{i}}$,

A69, page 6 of 8 
where $\varphi_{i}(s)$ is $\varphi(s) / \Gamma\left(b_{i}+B_{i} s\right)$. As a result, if all gamma functions $\Gamma\left(b_{i}+B_{i} s\right)$ only have single poles, we get as a series expansion,

$H_{p, q}^{m, n}\left[\begin{array}{c}(\boldsymbol{a}, \boldsymbol{A}) \\ (\boldsymbol{b}, \boldsymbol{B})\end{array} \mid z\right]=\sum_{i=1}^{m} \sum_{k=0}^{\infty} \frac{(-1)^{k}}{k ! B_{i}} \frac{\prod_{j=1, j \neq i}^{m} \Gamma\left(b_{j}-B_{j} \frac{b_{i}+k}{B_{i}}\right) \prod_{j=1}^{n} \Gamma\left(1-a_{j}+A_{j} \frac{b_{i}+k}{B_{i}}\right)}{\prod_{j=m+1}^{q} \Gamma\left(1-b_{j}+B_{j} \frac{b_{i}+k}{B_{i}}\right) \prod_{j=n+1}^{p} \Gamma\left(a_{j}-A_{j} \frac{b_{i}+k}{B_{i}}\right)} z^{\left(b_{i}+k\right) / B_{i}}$,

in agreement with Eq. (3.12) in Kilbas \& Saigo (1999). However, if several gamma functions share the same pole, then this pole is of second order, and the calculation becomes more cumbersome. Kilbas \& Saigo (1999) demonstrate that the Fox $H$ function can then be expressed as a logarithmic-power series rather than a simple power series. They present a generic expression valid for all orders of pole multiplicity. Here, we present a less general, but more explicit, series expansion in the case that two gamma functions $\Gamma\left(b_{j}+B_{j} s\right)$ share at least one pole. Without loss of generality, we can place these two gamma functions at the front, so that we can write

$\varphi(s)=\Gamma\left(b_{1}+B_{1} s\right) \Gamma\left(b_{2}+B_{2} s\right) \phi(s)$,

with

$\phi(s)=\frac{\prod_{j=3}^{m} \Gamma\left(b_{j}+B_{j} s\right) \prod_{j=1}^{n} \Gamma\left(1-a_{j}-A_{j} s\right)}{\prod_{j=m+1}^{q} \Gamma\left(1-b_{j}-B_{j} s\right) \prod_{j=n+1}^{p} \Gamma\left(a_{j}+A_{j} s\right)}$.

Now, suppose there is a tuple $\left(k_{1}, k_{2}\right)$ of indices so that $\beta_{1, k_{1}}=\beta_{2, k_{2}}$. The residue of this second-order pole is then, after some algebra,

$$
\begin{aligned}
\operatorname{Res}_{s=\beta_{1, k_{1}}}\left\{\varphi(s) z^{-s}\right\} & =\lim _{s \rightarrow \beta_{1, k_{1}}}\left\{\frac{\mathrm{d}}{\mathrm{d} s}\left[\left(s-\beta_{1, k_{1}}\right)^{2} \varphi(s) z^{-s}\right]\right\} \\
& =\phi\left(\beta_{1, k_{1}}\right)\left[-\ln z+B_{1} \psi\left(k_{1}+1\right)+B_{2} \psi\left(k_{2}+1\right)+\frac{\phi^{\prime}\left(\beta_{1, k_{1}}\right)}{\phi\left(\beta_{1, k_{1}}\right)}\right] \frac{(-1)^{k_{1}}(-1)^{k_{2}}}{\left(k_{1}\right) !\left(k_{2}\right) !} \frac{z^{-\beta_{1, k_{1}}}}{B_{1} B_{2}},
\end{aligned}
$$

with $\psi(s)=\Gamma^{\prime}(s) / \Gamma(s)$ the digamma function. Moreover, $\phi(s)$ is a product and quotient of gamma functions, so that $\phi^{\prime}(s) / \phi(s)$ can also be expressed as a sum of digamma functions. This means that all the machinery is available to express the Fox $H$ function as the rather daunting series expansion

$$
\begin{aligned}
H_{p, q}^{m, n}\left[\begin{array}{c}
(\boldsymbol{a}, \boldsymbol{A}) \\
(\boldsymbol{b}, \boldsymbol{B})
\end{array} \mid z\right]= & \sum_{i, k}^{\prime} \frac{(-1)^{k}}{k ! B_{i}} \frac{\prod_{j=1, j \neq i}^{m} \Gamma\left(b_{j}-B_{j} \frac{b_{i}+k}{B_{i}}\right) \prod_{j=1}^{n} \Gamma\left(1-a_{j}+A_{j} \frac{b_{i}+k}{B_{i}}\right)}{\prod_{j=m+1}^{q} \Gamma\left(1-b_{j}+B_{j} \frac{b_{i}+k}{B_{i}}\right) \prod_{j=n+1}^{p} \Gamma\left(a_{j}-A_{j} \frac{b_{i}+k}{B_{i}}\right)} z^{\left(b_{i}+k\right) / B_{i}} \\
& +\sum_{k_{1}}^{\prime \prime} \frac{(-1)^{k_{1}+k_{2}}}{k_{1} ! k_{2} ! B_{1} B_{2}} \frac{\prod_{j=3}^{m} \Gamma\left(b_{j}-B_{j} \frac{b_{i}+k_{1}}{B_{i}}\right) \prod_{j=1}^{n} \Gamma\left(1-a_{j}+A_{j} \frac{b_{i}+k_{1}}{B_{i}}\right)}{\prod_{j=m+1}^{q} \Gamma\left(1-b_{j}+B_{j} \frac{b_{i}+k_{1}}{B_{i}}\right) \prod_{j=n+1}^{p} \Gamma\left(a_{j}-A_{j} \frac{b_{i}+k_{1}}{B_{i}}\right)} z^{\left(b_{i}+k_{1}\right) / B_{i}}\left(C_{k_{1}}-\ln z\right)
\end{aligned}
$$

with the constants $C_{k_{1}}$ defined as

$$
\begin{aligned}
C_{k_{1}}= & B_{1} \psi\left(k_{1}+1\right)+B_{2} \psi\left(k_{2}+1\right)+\sum_{j=3}^{m} B_{j} \psi\left(b_{j}-B_{j} \frac{b_{1}+k_{1}}{B_{1}}\right)-\sum_{j=1}^{n} A_{j} \psi\left(1-a_{j}+A_{j} \frac{b_{1}+k_{1}}{B_{1}}\right) \\
& +\sum_{j=m+1}^{q} B_{j} \psi\left(1-b_{j}+B_{j} \frac{b_{1}+k_{1}}{B_{1}}\right)-\sum_{j=n+1}^{p} A_{j} \psi\left(a_{j}-A_{j} \frac{b_{1}+k_{1}}{B_{1}}\right) .
\end{aligned}
$$

The prime in the first summation in (A.10a) indicates that this sum covers only the single poles, and the double prime in the second summation indicates that this summation runs over the second-order poles. In the latter summation, we set $k_{2}=B_{2}\left(b_{1}+k_{1}\right) / B_{1}-b_{2}$.

\section{References}

Allen, P. D., Driver, S. P., Graham, A. W., et al. 2006, MNRAS, 371, 2

Andredakis, Y. C., Peletier, R. F., \& Balcells, M. 1995, MNRAS, 275, 874

Baes, M., \& Gentile, G. 2011, A\&A, 525, A136

Baes, M., \& van Hese, E. 2007, A\&A, 471, 419

Caon, N., Capaccioli, M., \& D'Onofrio, M. 1993, MNRAS, 265, 1013

Cardone, V. F. 2004, A\&A, 415, 839

Cellone, S. A., Forte, J. C., \& Geisler, D. 1994, ApJS, 93, 397

Ciotti, L. 1991, A\&A, 249, 99

Ciotti, L., \& Lanzoni, B. 1997, A\&A, 321, 724

Ciotti, L., \& Bertin, G. 1999, A\&A, 352, 447

Davies, J. I., Phillipps, S., Cawson, M. G. M., Disney, M. J., \& Kibblewhite, E. J. 1988, MNRAS, 232, 239

De Rijcke, S., Van Hese, E., \& Buyle, P. 2010, ApJ, 724, L171

D’Onofrio, M., Capaccioli, M., \& Caon, N. 1994, MNRAS, 271, 523

Elíasdóttir, Á., \& Möller, O. 2007, J. Cosmol. Astro-Particle Phys., 7, 6

Fox, C. 1961, Trans. Am. Mathem. Soc., 98, 395

Gadotti, D. A. 2009, MNRAS, 393, 1531

Gradshteyn, I. S., \& Ryzhik, I. M. 1965 (New York: Academic Press), 4th ed., ed. Yu. V. Geronimus, \& M. Yu. Tseytlin 
A\&A 534, A69 (2011)

Graham, A. W., \& Driver, S. P. 2005, PASA, 22, 118

Graham, A. W., \& Guzmán, R. 2003, AJ, 125, 2936

Haubold, H. J., Mathai, A. M., \& Saxena, R. K. 2007, Bull. Astron. Soc. India, 35, 681

Haubold, H. J., Mathai, A. M., \& Saxena, R. K. 2011, J. Comput. Appl. Math., 235, 1311

Kilbas, A. A., \& Saigo, M. 1999, J. Appl. Math. Stochast. Anal., 12, 191

Kilbas, A. A., \& Saigo, M. 2004, H-Transforms: Theory and Applications (CRC Press)

Mathai, A. M., \& Saxena R. M. 1978, The $H$ Function with Applications in Statistics and Other Disciplines (Wiley)

Mathai, A. M., Saxena R. M., \& Haubold H. J. 2009, The $H$-Function: Theory and Applications (Springer)

Mazure, A., \& Capelato, H. V. 2002, A\&A, 383, 384

Möllenhoff, C., \& Heidt, J. 2001, A\&A, 368, 16

Prugniel, P., \& Simien, F. 1997, A\&A, 321, 111

Sérsic, J. L. 1968, Cordoba, Argentina: Observatorio Astronomico

Srivastava, H. M., Gupta, K. C., \& Goyal, S. P. 1982, The H-Function of One and Two Variables with Applications (New Delhi, India: South Asian Publ.)

Trujillo, I., Graham, A. W., \& Caon, N. 2001, MNRAS, 326, 869

van Hese, E., Baes, M., \& Dejonghe, H. 2009, ApJ, 690, 1280 